\title{
Bacterium-Enabled Transient Gene Activation by Artificial Transcription Factor for Resolving Gene Regulation in Maize
}

Mingxia Zhao, ${ }^{\mathrm{a}, 1}$ Zhao Peng, ${ }^{\mathrm{b}, 1}$ Yang Qin, ${ }^{\mathrm{c}, 1}$ Ling Zhang, ${ }^{\mathrm{d}}$ Bin Tian, ${ }^{\mathrm{a}, 2}$ Yueying Chen, ${ }^{\mathrm{a}, 3}$ Yan Liu, ${ }^{\mathrm{c}}$ Guifang Lin, ${ }^{\mathrm{a}}$ Huakun Zheng, ${ }^{\mathrm{a}, 4}$ Cheng He, ${ }^{\mathrm{a}}$ Kaiwen Lv, ${ }^{\mathrm{e}}$ Harold N.Trick, ${ }^{\mathrm{a}}$ Yunjun Liu, ${ }^{\mathrm{c}}$ Myeong-Je Cho, ${ }^{\mathrm{f}}$ Sunghun Park, ${ }^{\mathrm{g}}$ Hairong Wei, ${ }^{\mathrm{d}}$ Jun Zheng, ${ }^{\mathrm{c}}$ Frank F. White, ${ }^{\mathrm{b}, 5}$ and Sanzhen Liu ${ }^{\mathrm{a}, 5}$

${ }^{a}$ Department of Plant Pathology, Kansas State University, Manhattan, KS 66506-5502, USA

${ }^{\mathrm{b}}$ Department of Plant Pathology, University of Florida, Gainesville, FL 32611-0680, USA ${ }^{c}$ Institute of Crop Sciences, Chinese Academy of Agricultural Sciences, Beijing 100081, P.R. China

${ }^{\mathrm{d}}$ College of Forest Resources and Environmental Science, Michigan Technological University, Houghton, MI 49931, USA

e State Key Laboratory of Tree Genetics and Breeding, Northeast Forestry University, Heilongjiang Harbin 150040, P. R. China

${ }^{f}$ Innovative Genomics Institute, University of California, Berkeley, CA 94704, USA

${ }^{9}$ Department of Horticulture and Natural Resources, Kansas State University, Manhattan, KS 66506-5502, USA

${ }^{1}$ These authors contributed equally to this work.

${ }^{2}$ Current address: Seeds Research, Syngenta Crop Protection, LLC, Research Triangle Park, North Carolina 27703

${ }^{3}$ Current address: DeltaMed Solutions, Inc., 220 Davidson Avenue, Suite 201, Somerset, NJ 08873

${ }^{4}$ Current address: National Engineering Research Center of JUNCAO Technology, College of Life Science, Fujian Agriculture and Forestry University, Fuzhou 350002, China

${ }^{5}$ Address correspondence to ffwhite@ufl.edu or liu3zhen@ksu.edu.

Keywords: transient activation, Xanthomonas, TALe, cuticular wax, maize

Running title: Bacterium-enabled gene activation in maize 


\section{ABSTRACT}

39 Cellular functions are diversified through intricate transcription regulations, and an understanding gene regulation networks is essential to elucidating many developmental processes and environmental responses. Here, we employed the TranscriptionalActivator Like effectors (TALes), which represent a family of transcription factors that are synthesized by members of the $\mathrm{Y}$-proteobacterium genus Xanthomonas and secreted to host cells for activation of targeted host genes. Through delivery by the maize pathogen, Xanthomonas vasicola pv. vasculorum, designer TALes (dTALes), which are synthetic TALes, were used to induce the expression of the maize gene glossy3 (g/3), a MYB transcription factor gene involved in the cuticular wax biosynthesis. RNA-Seq analysis of leaf samples identified $146 \mathrm{~g} / 3$ downstream genes. Eight of the nine known genes known to be involved in the cuticular wax biosynthesis were up-regulated by at least one dTALe. A top-down Gaussian graphical model predicted that $68 \mathrm{~g} / 3$ downstream genes were directly regulated by GL3. A chemically induced mutant of the gene Zm00001d017418 from the g/3 downstream gene, encoding aldehyde dehydrogenase, exhibited a typical glossy leaf phenotype and reduced epicuticular waxes. The bacterial protein delivery of artificial transcription factors, dTALes, proved to be a straightforward and powerful approach for the revelation of gene regulation in plants.

\section{INTRODUCTION}

59 Transcriptional regulation is essential for cellular differentiation and responses to environmental signals. Transcription factors (TFs) are key components for modulating gene expression and an understanding TF function is fundamental to elucidating gene regulation networks. Traditional approaches to transcription pathway analysis involve ectopic expression, in some cases transiently, and genetic mutation. Further analyses

64 involve the analysis of TF binding sites, including chromatin immunoprecipitation sequencing (ChIP-Seq) and the in vitro DNA affinity purification sequencing (DAP-Seq) (Bartlett et al., 2017). All of the approaches have advantages and limitations (Lai et al., 2019). Ectopic expression or knockouts are typically constructed to understand 
time, are not readily available or require considerable time to construct. Genome-wide transcriptional changes through the transient gene activation may offer another approach (Gleba et al., 2014). However, transient expression methods can be limiting, depending on species. Emerging nanomaterial technologies offer a potential option for delivery of nucleotides and proteins, and the techniques efficiently overcoming the barrier from plant cell walls are still evolving (Cunningham et al., 2018; Demirer et al., 2020).

Bacteria, principally pathogenic species, although not all, have evolved secretion systems to inject proteins into host cells to induce changes in the host metabolism, and facilitate colonization of host tissues (Costa et al., 2015; Deslandes and Rivas, 2012; Block et al., 2008). The type III secretion system (T3SS) is such a supramolecular complex that delivers bacterial proteins (effectors) to target cells (Green and Mecsas, 2016). Many plant pathogens of the genus Xanthomonas require a functioning T3SS for virulence and cause diseases on hundreds of plant species, including most major crop species (White et al., 2009; Büttner and Bonas, 2010). The Transcriptional-Activator Like effector (TALe) family is a group of type III effectors that have diverse functions for host cell manipulations, with the primary function of directing expression of specific disease susceptibility genes. The N-terminus of a TALe contains the T3SS secretion signal and the C-terminus processes domains for eukaryotic nuclear localization and transcription activation (Yang et al., 2000; Zhu et al., 1998; Van den Ackerveken et al., 1996). The central repetitive sequence consists of a variable number of repeats, each of which contains nearly identical 34-35 amino acid residues and variable di-residues (RVD) at the $12^{\text {th }}$ and $13^{\text {th }}$. The RVD of a repeat determines the specific recognition of a nucleotide base of four DNA nucleotides (Boch et al., 2009; Moscou and Bogdanove, 2009). The revelation of specific recognition between RVD and nucleotide bases provides a rationale for the construction of artificial, or designer, TAL effectors (dTALes) to target specific DNA sequences (Joung and Sander, 2013; Li et al., 2013b).

Here, we used TALe-mediated targeting activation of transcription as delivered by the maize pathogen Xanthomonas vasicola pv. vascularum (Xvv) (Perez-Quintero et al., 2020) to characterize the regulation of cuticular wax development. Cuticular waxes are derivatives of very-long-chain fatty acids (VLCFAs), which are produced through 
100 cyclic reactions that add two carbons per cycle (Kunst and Samuels, 2003; Lee and 101 Suh, 2013). Cuticular waxes are secreted through the plasma membrane to the plant 102 surface, providing a hydrophobic barrier to protect plants from water loss and other 103 environmental stresses (Fehling and Mukherjee, 1991). In maize, mutants with reduced 104 cuticular waxes can hold water droplets on leaves with water spraying, which is referred 105 to as the glossy phenotype. To date, more than 30 glossy loci have been discovered by 106 mutants, and at least 11 glossy genes were found to be responsible to glossy leaf 107 phenotype, including $g / 1, g / 2, g / 3, g / 4, g / 6, g / 8, g / 13, g / 14, g / 15, g / 26$, and cer8 (Tacke et 108 al., 1995; Li et al., 2019, 2013a; Liu et al., 2012; Moose and Sisco, 1996; Zheng et al., 109 2019; Hansen et al., 1997; Xu et al., 1997; Liu et al., 2009). Among them, the gl13 gene 110 encodes an $A B C$ transporter functioning in secretion of cuticular waxes through the 111 plasma membrane (Li et al., 2013a). The gl15 gene encodes an AP2-like TF, which

112 does not directly participate in the biosynthesis of cuticular wax but functions in juvenile113 to-adult transition of epidermal cells (Moose and Sisco, 1996). In this study, the artificial 114 transcription factors, or dTALes, were used to activate $g / 3$, an apparent early TF gene in 115 the biosynthesis of cuticular waxes, and identify the downstream genes of GL3 and 116 genes related to the cuticular wax pathway. 


\section{RESULTS}

120 A bacterium-enabled protein delivery system in maize

121 The Xvv strain Xv1601, a pathogen of maize, was used for delivery, and, based on

122 previous sequence analysis, is free of TALe genes (Perez-Quintero et al., 2020).

123 Phylogenetic analysis of 10 Xanthomonas species indicated that Xvv is genetically

124 close to Xanthomonas oryzae (Xo) (Supplemental Figure 1). Xv1601 does contain a

125 T3SS gene cluster that is syntenic with the clustered genes from a reference Xo strain

126 PXO99 ${ }^{A}$, which is known to deliver TALes during infection (Figure 1A). A knockout

127 mutant of $h r c C\left(h r c C^{-}\right)$, an essential gene of the T3SS, dramatically reduced the

128 virulence of $\mathrm{Xv1601}$, indicating T3SS is critical for the bacterial virulence (Figure 1B).

129 To test the ability of Xv1601 to deliver proteins to intact maize leaf cells, a plasmid

130 bearing a green enhanced fluorescent protein (eGFP) gene fused to the type III

131 secretion signal of AvrBs2 was constructed (Supplemental Figure 2) (Minsavage,

132 1990). To enhance detection, a nuclear localization signal was incorporated into the

133 protein (Khang et al., 2010). Upon inoculation of leaf tissue, GFP signals were detected

134 in host cells (Figure 1C). In this case, GFP was localized to the nucleus. 
A

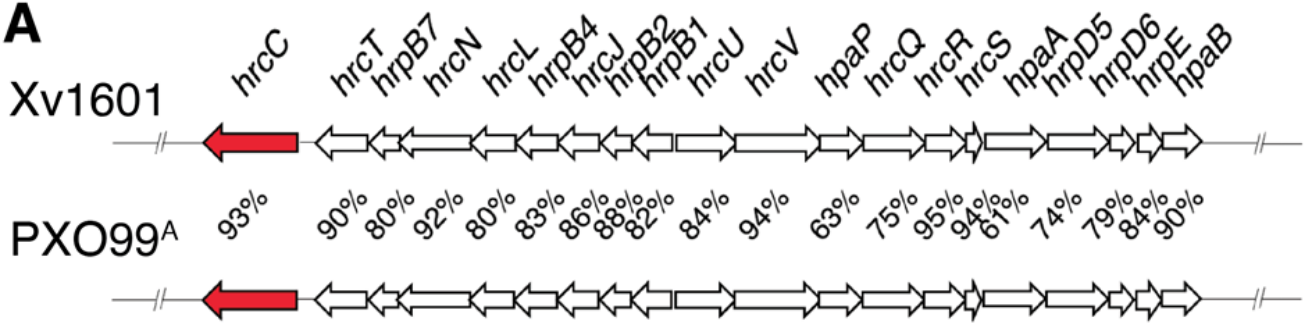

B

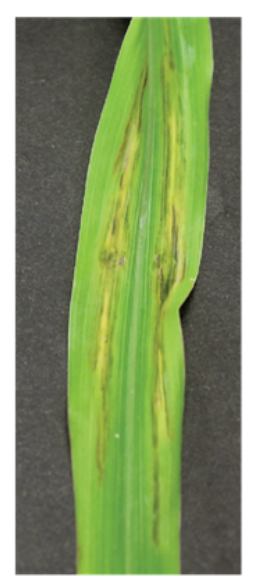

Xv1601

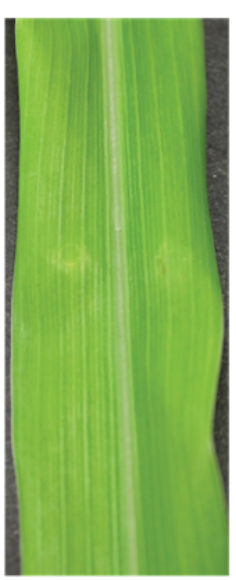

$\operatorname{hrcC}-$
C

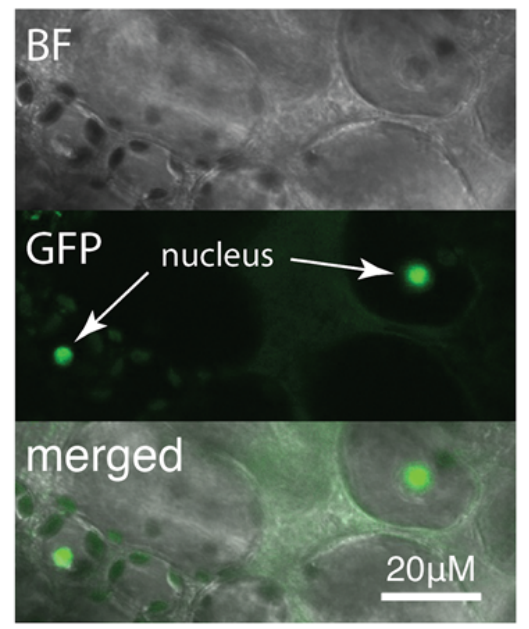

Figure 1. The T3SS of Xvv functions delivering proteins to intact cells

(A) Comparison of the type III secretion gene cluster of Xv1601 and the Xanthomonas oryzae strain PX099 ${ }^{A}$. The DNA identity between each orthologous pair is listed. The hrcC genes are highlighted in red. (B) Leaf phenotype five days after inoculation with the wildtype strain (Xv1601) and the hrcC knockout mutant strain. (C) Bright field (BF) and fluorescence (GFP) images of maize cells after $24 \mathrm{~h}$ of the infection with bacteria carrying a gene of AvrBs2::T3SS signal peptide-NLS::eGFP-NLS (Supplemental Figure 2). T3SS: The type III secretion system.

TALe-induced expression of a host gene

As Xv1601 does not contain any endogenous TALe genes, we tested the ability of the strain to deliver dTALes based on the ability to induce a targeted host gene. Two dTALes, referred to as dT1 and dT2, were constructed to target two non-overlapping 16-bp regions of the g/3 promoter (Figure 2A, 2B). Both dT1 and dT2 targeted regions are close to two predicted TATA boxes, which are $5 \mathrm{bp}$ and $48 \mathrm{bp}$ upstream of the transcription start site, respectively. Expression of g/3 was observed in seedling leaves. 
153 However, expression dropped to the undetectable levels by 14 days after planting

154 (Supplemental Figure 3). Therefore, 14-day seedlings were used to test for dTALes-

155 mediated induction of $g / 3$. Bacterial strains carrying either dT1 or dT2 activated $g / 3$

156 expression by $24 \mathrm{~h}$ after the bacterial inoculation (Figure 2C). Compared with dT2, dT1

157 promoted stronger induction of $g / 3$ as measured by quantitative reverse transcription

158 PCR (qRT-PCR) (Supplemental Figure 4). A time-series analysis of the gl3 expression

159 due to dT1 showed that relative expression reached 22 fold and 82 fold at $24 \mathrm{~h}$ and $48 \mathrm{~h}$

160 after inoculation, respectively, compared to the empty vector (Figure 2D).

A

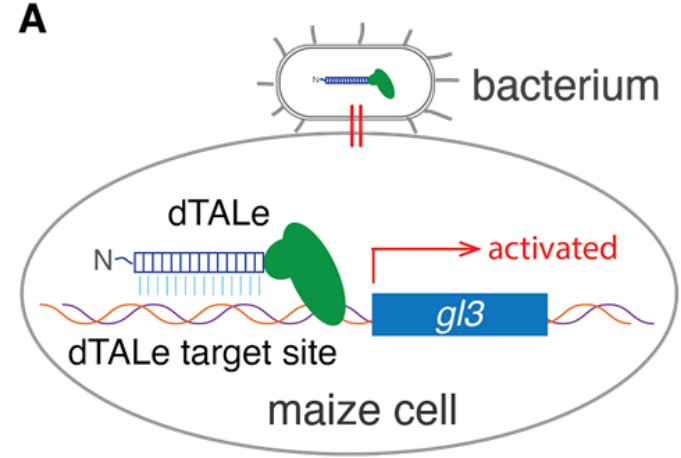

B

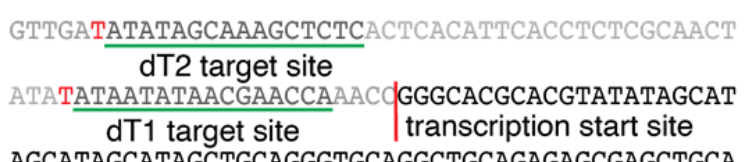
AGCATAGCATAGCTGCAGGGTGCAGGCTGCAGAGAGCGAGCTGCA

GCGCCATGGGCAGGCCGCCGTGCTGCGACAAGGAGGGGATC... translation start site
C

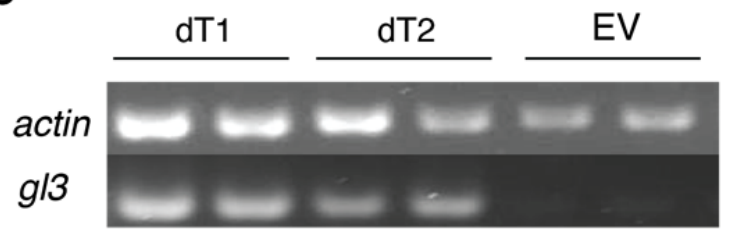

D

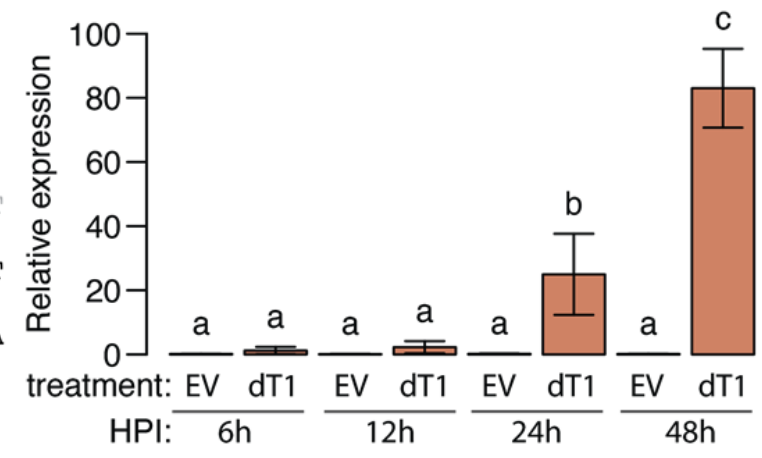

Figure 2. dTALe-dependent $g / 3$ gene expression

(A) Schematic of bacterium-mediated delivery of dTALes for the expression activation of maize g/3. (B) The target sequences for dT1 and dT2 (underlined in green). The transcription start site is indicated by a vertical red line. The translation start site ATG for GL3 is underlined in red. (C) Semi-RT-PCR of the g/3 expression from 14-day old seedling leaves. Treatments with two replicates are shown for bacteria carrying either $\mathrm{dT} 1, \mathrm{dT} 2$, or the empty vector (EV). The constitutively expressed actin gene was used for loading controls. (D) qRT-PCR of the g/3 expression at $6,12,24$, and 48 hours post

171 inoculation (HPI). The bar heights are the average of three biological replicates per treatment per time points. Error bars represent standard deviation. Values with the 
173 same letter do not differ at the significance level of 0.05 as determined by ANOVA and

174 Tukey's honestly significant difference.

\section{GL3 downstream genes identified through RNA-Seq}

177 To determine the genes regulated by GL3, RNA-Seq was performed using tissues after 178 treatments with bacteria carrying dT1, dT2, and the empty vector (EV). The basal 179 expression level of $g / 3$ in young leaves was low, while treatments with dT1 or dT2 180 exhibited 191 and 74 fold induction of $g / 3$, respectively (Figure 3A, Table 1). The comparison of dT1 with the EV control identified 1,249 differentially expressed genes (DEGs) at the false discovery rate (FDR) of 5\%, among which 499 were up-regulated. A comparison of dT2 versus EV resulted in 430 DEGs were identified at the FDR of $10 \%$, of which 156 were up-regulated (Figure 3B). Note that a higher FDR value used in dT2 is due to a lower level induction of the g/3 expression. The 92 common up-regulated DEGs of dT1 and dT2 and 54 common down-regulated DEGs were deemed as the $g / 3$ downstream genes (excluding g/3). Gene ontology (GO) analysis showed that the genes related to fatty acid biosynthesis and the endoplasmic reticulum (ER) are overrepresented in the 92 up-regulated genes (Figure 3C).

Of nine known glossy genes, which do not include g/3 or gl15, six were among the 92 up-regulated DEGs that were up-regulated by both dTALes, and additional two genes were only up-regulated by dT1 (Table 1). All eight genes showed the same regulation pattern by two dTALes, of which dT1 exhibited a stronger induction (Figure 3D). The only glossy gene that was unaffected by the $g / 3$ induction is $g / 13$, which is an $A B C$ transporter functioning in the secretion of cuticular waxes across the plasma membrane (Li et al., 2013a). Besides the known glossy genes, 86 additional genes were up-regulated by the dTALes including six genes encoding 3-ketoacyl-CoA synthases (KCS) as gl4 does (Liu et al., 2009), two genes encoding HXXXD-type acyl199 transferase related proteins similar to gl2 (Tacke et al., 1995), three genes encoding 200 GDSL esterase/lipase proteins, which was reported to be involved in wax biosynthesis 201 (Tang et al., 2020), and two genes encoding aldehyde dehydrogenases (Supplemental

202 Data Set 1). The 54 down-regulated genes were identified in both dT1 and dT2 comparisons with the EV group, which do not include any known glossy genes. Most 
204 glossy genes were previously reported to be clustered in a turquoise module of a gene 205 co-expression network (GCN295) constructed using 295 RNA-Seq data (Zheng et al., 206 2019). Of the 92 genes up-regulated by gl3, 61 are present in GCN295, and 38/61 were 207 assigned to the turquoise module. In contrast, only three genes from the 54 genes 208 down-regulated by dTALes are in the turquoise module. Collectively, from the RNA-Seq 209 results, the $\mathrm{g} / 3$ gene appeared to be a master regulator positively modulating 210 biosynthesis of cuticular waxes.

A conventional neural network (CNN) deep learning approach was used to 212 determine the probability that a gene is regulated by GL3 from 739 publicly available 213 RNA-seq datasets of inbred line B73. To train the prediction model, the gene pairs of

214 TFs and their targeted genes mapped from Arabidopsis gene regulation data were used 215 as the positive pairs (Yilmaz et al., 2011), and the random gene pairs that did not 216 overlap with positive pairs were used as the negative control pairs. The deep learning 217 predicted that $59.6 \%$ GL3 downstream genes were regulated by GL3 with a probability 218 of at least 0.8 , while $17.7 \%$ of the 594 control genes that were unaffected by both dT1 219 and dT2 were predicted (Supplemental Table 1, Supplemental Data Set 2). The in 220 silico prediction supported that most of the $g / 3$ downstream genes revealed are 221 regulated by $g / 3$.

\section{Probability-based identification of GL3 directly regulated genes}

224 The GL3 downstream genes are either directly or indirectly regulated by GL3. A topdown Gaussian graphical model (GGM) algorithm was employed to find the genes that were likely to be directly regulated by GL3 (Lin, Li et al. 2013, Wei 2019, Wei, Liu et al.

227 2020). From the 146 GL3 downstream genes, the algorithm first identified 93 GL3

228 responsive genes that had high concordance in expression levels with $\mathrm{gl3}$ expression 229 with the expression data from the dT1, dT2, and EV RNA-seq experiments 230 (Supplemental Data Set 3). The expression data of g/3 and the 93 GL3 responsive 231 genes were then used to infer the directly regulated genes of GL3. Briefly, two genes 232 from the GL3 responsive genes were combined with g/3 to form a triple gene block for 233 evaluation. If the corrected $p$-value with multivariate delta method (Methods) for each 234 triple gene block is less than 0.05, g/3 was scored as to interfere with the two 
235 responsive genes once. All triple gene blocks were evaluated, and the interference

236 frequency for each gene was calculated. As a result, 68 genes that were interfered by

$237 \mathrm{~g} / 3$ were identified as direct targets of GL3, including 60 up-regulated and 8 down-

238 regulated genes (Figure 3E). The remaining 78 genes from 146 GL3 downstream

239 genes are likely to be indirectly regulated by GL3 (Supplemental Data Set 3). Five

240 glossy genes that were associated with dT1 and dT2, namely, gl1, gl4, gl6, gl26, and

241 cer8, were predicted to be directly regulated by GL3, indicative of the direct regulation

242 role of GL3 in biosynthesis of cuticular waxes.

243

244
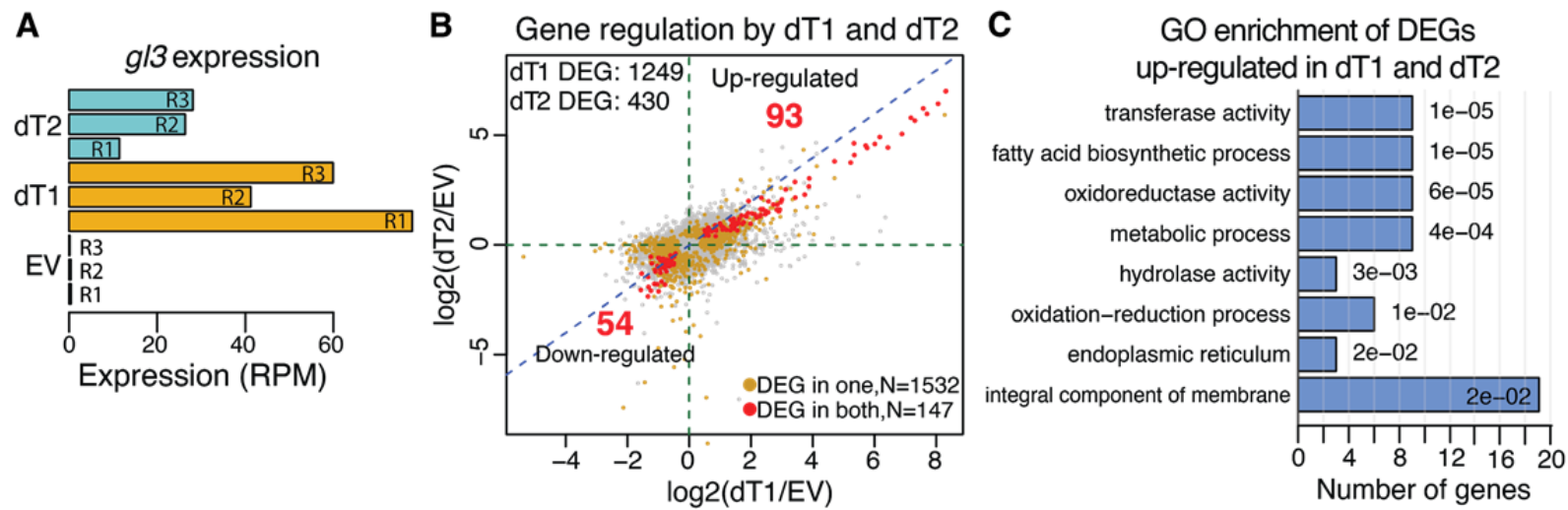

D Expression of other glossy genes

E

DEG ( $\mathrm{N}=146)$
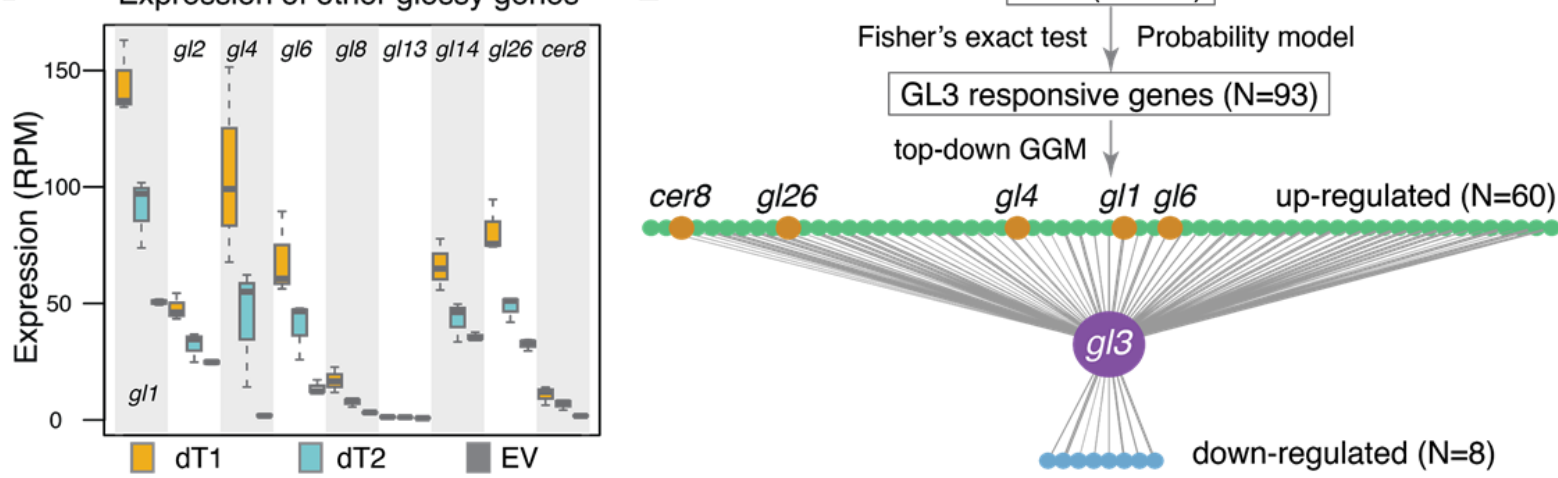

Figure 3. Gene expression associated with TALe-dependent expression of $g / 3$.

247 (A) Expression in RPM (reads per million reads) of g/3 from RNA-Seq data. R1-R3

248 represent biological replicates. The treatment groups EV, dT1, dT2 stand for constructs

249 of empty vector, dT1, and dT2, respectively. (B) Scatter plot between log2 fold changes

250 of gene expression in the comparison of dT1 versus EV and that in the comparison of

251 dT2 versus EV. The 93 genes up-regulated by both dT1 and dT2 include g/3. Gray, 
252 orange, and red points represent unaffected, DEGs in one comparison, and DEGs in

253 both comparisons, respectively. (C) Gene ontology (GO) enriched in the DEGs in both

254 comparisons. Numbers besides bars are p-values of GO enrichment tests. (D)

255 Expression in RPM of nine glossy genes that affect cuticular wax accumulation in three

256 treatment groups. (E) Direct regulation by GL3 indicated by the top-down GGM

257 analysis. The upper layer listed up-regulated genes by g/3 and the bottom layer listed

258 down-regulated genes. The thickness of connection lines represents the number of

259 interferences by GL3 for each gene. Glossy genes are highlighted in orange.

260

261 Table 1. Differential expression of known glossy genes

\begin{tabular}{|c|c|c|c|c|c|c|c|}
\hline \multirow{2}{*}{ Gene } & \multirow{2}{*}{$\begin{array}{c}\text { Gloss } \\
y\end{array}$} & \multicolumn{3}{|c|}{ dT1 vs. ev } & \multicolumn{3}{|c|}{ dT2 vs. ev } \\
\hline & & Up fc ${ }^{*}$ & qvalue & significant & Up fc ${ }^{*}$ & qvalue & significant \\
\hline Zm00001d020557 & $g / 1$ & 2.87 & $3.74 \mathrm{E}-32$ & yes & 1.91 & 2.57E-07 & yes \\
\hline $\mathrm{Zm00001d002353}$ & $g / 2$ & 1.94 & $1.26 \mathrm{E}-10$ & yes & 1.38 & 0.208 & no \\
\hline Zm00001d052397 & $g / 3$ & 191.2 & 4.57E-89 & yes & 74.31 & $1.46 \mathrm{E}-18$ & yes \\
\hline Zm00001d051787 & $g / 4$ & 52.53 & 4.24E-95 & yes & 22.99 & 1.37E-08 & yes \\
\hline Zm00001d041578 & $g / 6$ & 4.96 & $1.59 E-25$ & yes & 3.08 & $3.20 \mathrm{E}-08$ & yes \\
\hline Zm00001d017111 & $g / 8$ & 5.51 & $3.28 E-20$ & yes & 2.63 & 1.61E-05 & yes \\
\hline Zm00001d039631 & $g / 13$ & 1.57 & 0.482 & no & 1.40 & 0.906 & no \\
\hline Zm00001d004198 & $g / 14$ & 1.85 & 5.62E-07 & yes & 1.28 & 0.481 & no \\
\hline Zm00001d008622 & $g / 26$ & 2.52 & $1.25 \mathrm{E}-20$ & yes & 1.58 & 2.82E-04 & yes \\
\hline $\mathrm{Zm00001d024723}$ & cer8 & 5.93 & $6.13 \mathrm{E}-15$ & yes & 3.96 & $5.80 \mathrm{E}-08$ & yes \\
\hline
\end{tabular}

${ }^{*}$ up-regulated fold change

\section{A g/3 downstream gene functions in cuticular wax accumulation}

265 Due to the presence of most known glossy genes in the DEGs up-regulated by $g / 3$, the

266 dTALe up-regulated genes may contain unknown genes involved in biosynthesis of

267 cuticular waxes. The genes that were up-regulated by both dTALes and assigned to the

268 turquoise module of GCN295 were selected as the candidate glossy genes for the

269 validation. Ethyl methanesulfonate (EMS) induced mutants of four candidate genes

270 were obtained from a maize EMS mutant stock collection (Lu et al., 2018). All mutants

271 were screened for the glossy phenotype. No glossy phenotype was observed for

272 mutants carrying premature stop codons in the three genes Zm00001d046642,

273 Zm00001d028241, and Zm00001d032719, which encode GDSL esterase/lipase, 3- 
274 ketoacyl-CoA synthase, and long-chain-alcohol oxidase FAO4B, respectively

275 (Supplemental Table 2). Zm00001d017418, which encodes aldehyde dehydrogenase,

276 is up-regulated by treatments with either dT1 or dT2 (Figure 4A). The EMS mutant

277 (ems4-12ff6f) with a premature stop codon in the second exon of Zm00001d017418

278 displayed a glossy phenotype, indicating reduced accumulation of cuticular waxes

279 (Figure 4B, 4C). Total leaf waxes on ems4-12ff6f mutants were reduced by $\sim 40 \%$ of

280 that in the wildtype (Figure 4D). Microscopic examination of wax components on the

281 leaf surface revealed fewer wax crystals accumulated on leaf surfaces of mutant lines

282 as compared to wildtypes (Figure 4E). Wax component analysis found a decrease in

283 C30 and longer chain primary alcohols, alkanes, and fatty acids (Supplemental Figure

$2845)$.

285

A

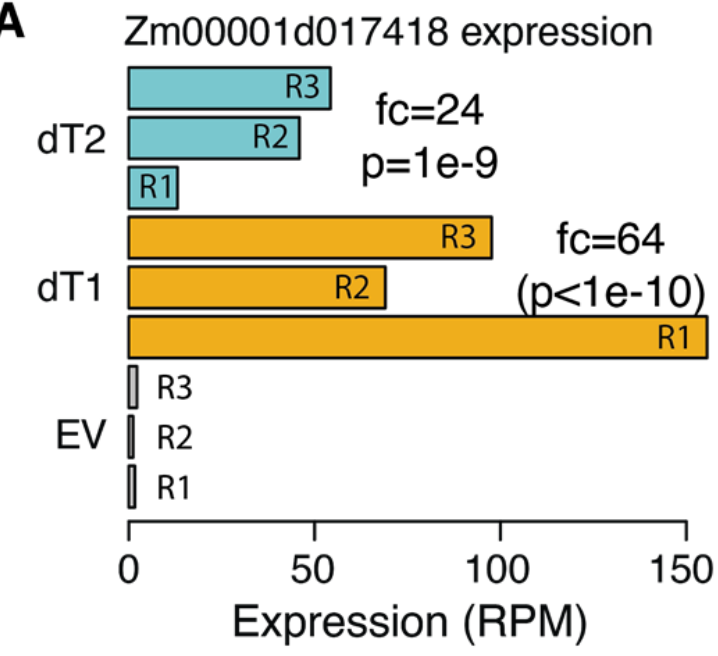

B

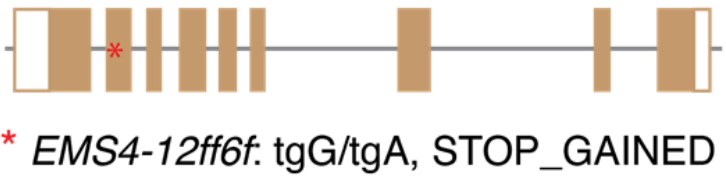

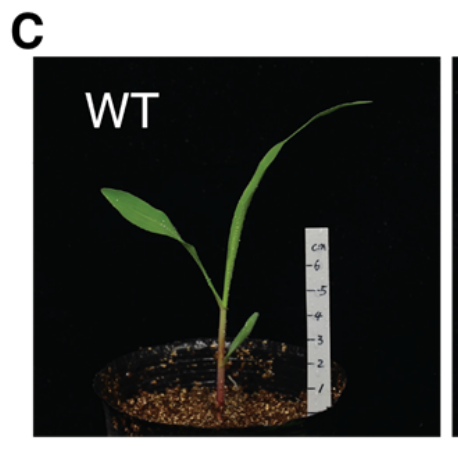

D

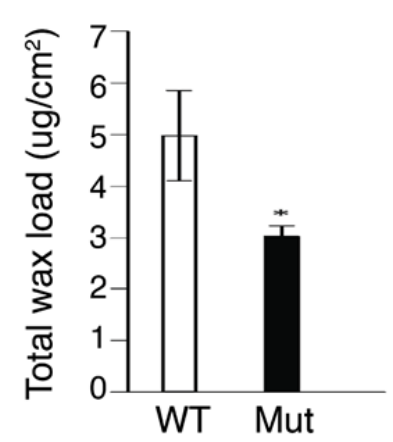

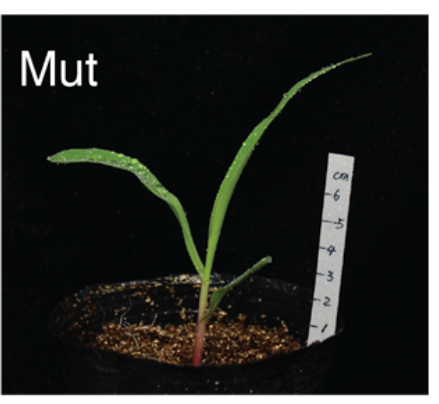

$\mathbf{E}$

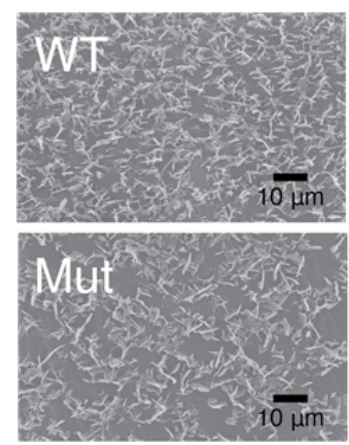

Figure 4. A new glossy gene Zm00001d017418.

288 (A) Expression of the candidate gene Zm00001d017418. R1-R3 represent biological 289 replicates. The treatment groups EV, dT1, dT2 stand for constructs of empty vector, 290 dTALe 1, and dTALe 2, respectively. fc, fold change in expression relative to EV; $p$, 291 adjusted p-value from RNA-Seq analysis. (B) Gene structure of the isoform of 
292 Zm00001d017418_T001. Boxes are exons and blank boxes represent untranslated 293 regions. Start points at the EMS mutation location, which produces a premature stop 294 codon. (C) The visible glossy phenotype of the EMS mutant and the wildtype (WT). 295 Water drops were present on the surfaces of mutant seedling leaves due to reduced 296 epicuticular waxes. (D) Total cuticular wax loads and wax components of mutants and 297 wildtypes. (E) Epicuticular wax contents on the leaf in the wildtype and the mutant 298 detected via scanning electron microscopy (SEM, x10,000 magnification). 


\section{DISCUSSION}

302 Here, the maize pathogen Xvv and the bacterial T3SS system were used for protein delivery into intact maize cells and, in this specific case, characterization of the cuticular wax pathway. Although considered destructive of plant tissue, Xanthomonas species are best considered as hemi-biotrophic in that the pathogens interact with intact cells for some time before destruction of the cells is evident in compatible interactions. In the initial demonstration, the T3SS signal of the effector AvrBs2 was used to direct GFP to intact cells. NLS was added to the effector to concentrate the protein in nuclei, both as evidence for intact cellular organelle and to facilitate detection of the protein in plant cells. For a demonstration of the utility, the approach was used to study consequences of ectopic expression of the MYB TF GL3 through induction by synthetic TALe, or dTALe, transcription factors. TALe effectors are particularly useful for the approach as TALes already have T3SS secretion signal sequences and NLS for localization into host cell nuclei. Although Xvv does not contain endogenous TALes, the presence of TALes with biological function in disease, including TALes that target host TFs, in closely related strains indicated that TALe delivery would be successful. Previous experience with the so-called American strains of $\mathrm{Xo}$, which also lack endogenous TALe genes, has indicated that TALes can be delivered by TALe-deficient strains (Tran et al., 2018). In this study, two dTALes were targeted to two separate DNA sequences in the promoter of $g / 3$. Both dTALes resulted in $g / 3$ induction as shown by both qRT-PCR and RNA-Seq. In addition to expression of $g / 3$, evidence was obtained that GL3-regulated genes were identified as a consequence of dTALe activation of $g / 3$.

323 The best evidence is that one of the apparent GL3 downstream genes,

$324 Z m 00001 d 017418$, was up-regulated and has the glossy phenotype, which results in reduced wax deposition on leaves, when mutated. The failure of displaying a glossy

326 phenotype of mutants from three other genes does not indicate no involvement in GL3-

327 dependent events and might be due to the functional redundancy in the maize genome.

328 In addition, GL3 downstream genes include most known glossy genes. The results

329 indicate the master regulatory role of GL3 in biosynthesis of cuticular waxes, and 330 provide strong evidence for the efficacy of the dTALe system for revelation of gene 331 regulations. In the future, experimental data can be generated to further examine the 
332 binding motif of the TF GL3. Also, given the fact that the g/3 gene is largely silenced at 333 the adult stages (Zheng et al., 2019), it would be interesting to examine the impacts on 334 the wax biogenesis from the constitutive expression of $g / 3$. More genes, particularly TFs downstream of GL3, could be examined through dTALe activation and/or knockouts, to further understand the regulation network of cuticular wax biosynthesis.

The dTALe activation system is easy to manipulate, and Xanthomonas strains are easy to culture. Besides the simplicity of the system, it is flexible to control the bacterial load by adjusting the concentration and amount of bacterial inoculum. At the same time, limitations need to be considered for the experimental design. First, multiple independent dTALes are needed to reduce the impacts of off-targeting gene induction. Multiple dTALes help discriminate between off-target gene induction with the idea that independent binding sites will not result in induction of the same off-target genes. Given that no specific domain other than a "T" preceding the binding site is required (Moscou and Bogdanove, 2009), candidate dTALe binding sites are relatively abundant. Second, bacterial infection and other T3SS effectors could potentially interfere with host gene expression or host protein function, if related to defense responses. The bacterium carrying an empty vector as the control, as implemented in this study, should largely reduce the impacts from bacteria.

The downstream genes of a dTALe targeted gene include direct or indirect targets of the dTALe binding gene. Based on expression patterns, direct and indirect 352 regulations are distinguishable with dedicated computational algorithms. The top-down 353 GGM algorithm, with the input of a short time-course data, had been shown to separate 354 the directly from indirectly regulated genes with more than 90\% accuracy (Wei, 2019; 355 Lin et al., 2013) and about 80\% accuracy for RNA-Seq data from stably transgenics lines (Wei et al., 2020). In this study, no time-course data were generated. However, the

357 variation of $g / 3$ expression induction within a dTALe treatment, probably due to the 358 variation in the bacterial amount injected during inoculation, and between two dTALes 359 mimics multiple levels of $\mathrm{gl3}$ induction as in time-course experiments. The data, 360 therefore, enabled the top-down GGM algorithm to identify the genes that closely 361 followed expression changes of $g / 3$, which were deemed to be directly regulated genes. Alternatively, the result from dTALe experiments could be combined with the results 
363 from DAP-Seq or CHIP-Seq that examines protein-binding sites to identify direct

364 targets.

365 The Xanthomonas bacteria can be used as a general tool for protein delivery to 366 plant host cells. Xanthomonas bacterial strains are available for most crops and have a 367 well-documented ability to deliver diverse proteins. This gene activation through dTALes 368 represents a unique system to study transcriptional regulation. The protein delivery 369 system can also be utilized to study plant-pathogen interactions. For example, any 370 effector gene can be engineered to the Xanthomonas bacterium and delivered to host 371 cells for examining defense responses. To reduce pathogenic effects from

372 Xanthomonas, the bacterium can be modified for the reduced host cell toxicity and a 373 higher capacity for protein delivery. 
METHODS

\section{Genetic materials}

378 The bacterium Xv1601 is pathogenic on maize (Perez-Quintero et al., 2020). A hrcC

379 knockout mutant was generated following protocol previously described (Peng et al., 380 2016). The maize inbred line A188 (PI 693339) were obtained from the North Central 381 Regional Plant Introduction Station and maintained at Kansas State University. Plants 382 were grown in a growth chamber under $27^{\circ} \mathrm{C}$ during daytime and $21^{\circ} \mathrm{C}$ at night with 16 383 hours of photoperiod. EMS mutants were ordered from the Maize EMS induced Mutant 384 Database (MEMD) (Lu et al., 2018).

\section{Design and assembly of protein delivery constructs}

387 The pENTR ${ }^{\text {TM }} 11$ Dual Selection Vector (Thermo Fisher Scientific, USA) was digested by Kpnl and Xhol, and the DNA fragments containing AvrBs2 promoter, the type III signal peptide, and eGFP were cloned into the digested plasmid according to NEBuilder HiFi DNA Assembly protocol (New England Biolabs, USA). The assembled entry construct was then cloned into the broad host-range vector pHM1 by the Gateway cloning (Supplemental Figure 2) and transformed into Xv1601 strain by electroporation using Bio-rad Micropulser (Peng et al., 2019).

\section{Design and assembly of dTALe}

396 The promoter elements targeted by TAL effectors are typically, not far away, upstream 397 of transcriptional start sites (Moscou and Bogdanove, 2009). Based on previous reports, 398 most TAL effectors (e.g., PthA4, AvrBs3, PthXo2, PthXo3, AvrXa7, PthXo6 and PthXo7) 399 binded at TATA box regions while some (e.g., PthXo1 and Tal8) targeted the regions a 400 few base pairs upstream of TATA boxes (Kay et al., 2007; Sugio et al., 2007; Antony et 401 al., 2010; Hu et al., 2014; Zhou et al., 2015; Peng et al., 2019). The two dTALes, dT1 402 and dT2, were designed to specifically target a TATA box region and an upstream 403 region of the TATA box in the promoter of $g / 3$, respectively. In addition, A "T" preceding 404 each dTALe binding element was required (Moscou and Bogdanove, 2009). The 405 Golden Gate TALEN assembly protocol was followed to construct the two dTALes 406 (Cermak et al., 2011). Briefly, the kit (Golden Gate TALEN and TAL Effector Kit 2.0) 
consisting of 86 library vectors was ordered from Addgene (www.addgene.org). To assemble the dTALe harboring 16 repeats, first 10-repeat TAL array and second 5repeat TAL array were constructed into the destination vectors pFUS_A and pFUS_B5, respectively. The resultant vectors, the last-repeat plasmid, and the destination vector pTAL1 were digested with Esp3I restriction enzyme (Thermo Fisher Scientific, USA) and ligated with T4 Ligase (New England Biolabs, USA) to fuse all TAL repeat arrays into the pTAL1 destination vector. The dTALes were then cloned into the broad hostrange vector pHM1 and transformed into Xv1601 strain by electroporation using Bio-rad Micropulser (Peng et al., 2019).

\section{Bacterial culture and inoculation}

Xv1601 bacteria were grown on tryptone sucrose agar medium at $28^{\circ} \mathrm{C}$ (Peng et al., 2016). The bacterial inoculum was prepared with the $O D_{600}$ range from 0.2 to 0.3 in the PBS buffer for plant inoculation. The second leaf of 14-day seedlings of the inbred line A188 was inoculated with the needleless syringe infiltration method. Approximately six centimeters of the second leaf from $2 \mathrm{~cm}$ away from the tip to the leaf base was filled with bacterial solution.

\section{Quantitative RT-PCR for quantifying g/3 expression at multiple seedling stages}

Shoot or second-leaf samples from A188 seedlings were collected from 3, 4, 5, 8, 14 days after seed germination. RNA was extracted from sampled tissues with Qiagen RNeasy Plant Mini Kit (Qiagen, Germany) and treated with DNase (Qiagen, Germany) to remove DNA contamination. First-strand cDNA was synthesized using Thermo Scientific Verso cDNA Kit (Thermo Fisher Scientific, USA) with anchored oligo dT primers. Quantitative RT-PCR was performed with g/3 specific primers (Supplemental Table 3) and $\mathrm{iQ}^{\mathrm{TM}} \mathrm{SYBR}^{\circledR}$ Green Supermix (BioRad, USA) and conducted on a BioRad CFX with 96-well reaction blocks under the following PCR conditions: $95^{\circ} \mathrm{C}$ for $3 \mathrm{~min}$, followed by 40 cycles of $15 \mathrm{~s}$ at $95^{\circ} \mathrm{C}$ and $30 \mathrm{~s}$ at $55^{\circ} \mathrm{C}$. The Actin gene with the actin primers (Supplemental Table 3) was used as a reference gene to normalize g/3 expression levels. The mean cycle threshold values $(\mathrm{Ct})$ from technical replicates were used to calculate relative gene expression. The relative $g / 3$ expression was determined using the formula $100 \times 2^{\wedge}\left(\mathrm{Ct}_{\mathrm{actin}}-\mathrm{Ct}_{\mathrm{g} \mid 3}\right)$, where $\mathrm{Ct}_{\mathrm{actin}}$ and $\mathrm{Ct}_{\mathrm{gl} 3}$ represent the $\mathrm{Ct}$ values of 
actin and $g / 3$, respectively.

441 To examine the expression induction of two dTALes, the second leaf of 14-day 442 seedlings were inoculated with the bacteria containing dT1, dT2, and EV. Inoculated 443 leaf tissues except for the inoculation position were collected at $24 \mathrm{~h}$ post inoculation.

444 Three plants with the same treatment were pooled in a tissue sample. The bacterium 445 with dT1 was used to examine the expression induction at multiple time points after the 446 inoculation of the bacterium. Three biological replicates were conducted with three 447 plants in each group. The inoculated leaf tissues were collected at $6 \mathrm{~h}, 12 \mathrm{~h}, 24 \mathrm{~h}$, and $44848 \mathrm{~h}$ post inoculation. qRT-PCR as mentioned was employed for the quantification of 449 gl3 expression.

\section{RNA-Seq experiment and data analysis}

452 An RNA-Seq experiment was performed to understand the $\mathrm{g} / 3$ downstream gene 453 regulation. The bacterial inoculum was prepared to the $0.2-0.30 \mathrm{OD}_{600}$ range in the PBS 454 buffer. The second leaf of 14-day old seedlings were inoculated with a needleless 455 syringe infiltration method. Three biological replicates (R1, R2, R3) were conducted for 456 each of three treatment groups of which bacteria separately carried dT1, dT2, EV 457 (empty vector). Inoculated leaf tissues were collected at 24 hours post inoculation. 458 Three plants with the same treatment were pooled in a tissue sample. As a result, nine 459 tissue samples were collected in total. RNA were extracted from sampled tissues with 460 Qiagen RNeasy Plant Mini Kit. Sequencing libraries were prepared and sequenced on a 461 Novaseq 6000 Illumina platform at Novogene Inc.. Adaptor sequences and low-quality 462 bases of raw reads were trimmed by Trimmomatic (version 0.38) (Bolger et al., 2014). 463 Trimmed reads were aligned to the B73 reference genome (B73Ref4) using STAR 464 (2.7.3a) (Dobin et al., 2013). Uniquely mapped reads were used for counting reads per 465 gene. DESeq2 (version 1.26.0) was used to identify differentially expressed genes 466 between each of the two dTALe groups (dT1 and dT2) and the EV group. Multiple tests 467 were accounted for by the false discovery rate (FDR) method with the FDR cutoffs of $4685 \%$ for dT1 and 10\% for dT2 (Benjamini and Hochberg, 1995). 


\section{Glossy phenotyping}

471 The glossy phenotype was identified by spraying water on seedlings at the two or three 472 leaf stage. Seedlings whose leaves were covered with small water droplets were 473 identified as glossy mutants.

\section{Scanning electron microscopy (SEM)}

476 The second leaves collected from ems4-12ff6f mutant and wildtype plants were used for scanning electron microscopy analysis (HITACHI, Japan) (Aharoni et al., 2004).

\section{Analysis of wax composition}

480 Wax extraction and gas chromatography-mass spectrometry (GC-MS) analyses were performed according to the described methods with some modifications (Chen et al., 2017). The ems4-12ff6f mutant and wildtype plants were grown in the substrate of roseite and sand (1:1) at a growth chamber $(25 \square)$ until the three-leaf stage. The second leaves (about $300 \mathrm{mg}$ ) were collected and immersed in $3 \mathrm{~mL}$ of chloroform for $1 \mathrm{~min}$, which dissolved $15 \mu \mathrm{g}$ nonadecanoic acid (C19) as internal standards. The solvents were transferred into new vials and evaporated under a gentle stream of nitrogen gas.

487 The residue was derivatized with $100 \mu \mathrm{L}$ of $\mathrm{N}$-methyl-N-(trimethylsilyl) trifluoroacetamide 488 and incubated for $1 \mathrm{~h}$ at $50 \square$. These derivatized samples were then analyzed by GC-MS 489 (Agilent gas chromatograph coupled to an Agilent 5975C quadrupole mass selective 490 detector). The area of leaves was calculated by IMAGEJ software 491 (http://imagej.nih.gov/ij/). The amount of leaf wax was related to unit surface area.

\section{Prediction of $g / 3$ regulated genes through deep learning}

494 In total, 739 B73 paired-end RNA-Seq data from diverse tissues and treatments were 495 collected from the NCBI Sequence Read Archive (SRA) database (Supplemental Data 496 Set 4). Software Trimmomatic (version 0.38) (Bolger et al., 2014) was used to trim the 497 adaptor sequence and low-quality bases of raw reads. Remaining paired-end reads 498 were aligned to B73 reference genome (B73Ref4) (Jiao et al., 2017) using STAR 499 (version 2.6.0) requiring concordant mapping positions of paired-end reads (Dobin et 
500 al., 2013). Raw read counts per gene were calculated by STAR and then normalized by 501 the library sizes of RNA-Seq samples to represent gene expression.

502 The 2,140 pairs of TFs and their putative targeted gene in maize obtained by 503 homologous mapping of Arabidopsis experimented verified regulatory gene pairs from 504 the Arabidopsis Regulatory Network database (Yilmaz et al., 2011) were used as the 505 positive training data set for deep learning. To generate a negative data set, we 506 randomly generated 2,140 gene pairs that do not contain above positive relationships. 507 The maize transcriptomic data of these 4,280 gene pairs used for training the 508 convolutional neural networks (CNN) model for predictions. The input data set of these 5094,280 pairs of genes were from 739 B73 RNA-seq data. Therefore, the data set 510 contains 4,280 gene pairs and each gene has 739 features. 428 gene pairs and their 511 expression data, which account for $10 \%$ of the whole data set, were randomly drawn 512 and used as the validation data set. The test data set, which contains $\mathrm{g} / 3$ versus $146 \mathrm{~g} / 3$ 513 downstream genes, and also g/3 versus 594 g/3-unaffected genes, were extracted from 514 the 739 RNA-Seq data set.

Besides expression data, two additional dimensions, the product and the 516 absolute difference of each pair of two genes, g/3 and a putative target gene, were 517 calculated and added as additional features. We employed Keras and TensorFlow 518 libraries to develop the convolutional neural networks (CNN) using $R$ libraries. The 519 architecture of the CNN includes three parts: the input, feature extractor and classifier. 520 The feature extractor contains several building blocks, each containing a convolution 521 layer and a pooling layer. A convolution layer consists of multiple filters that help identify 522 features, and activation functions that are to convert linear input to non-linear output. A 523 pooling layer provides the down-sampling operation to reduce the dimensions of the 524 feature map. The classifier is made up of a flatten layer and several fully connected 525 (FC) layers and each FC layer is followed by an activation function. The flatten layer 526 takes the results from feature extractor process and flatten them into a single long 527 vector that can be an input for the next FC layer, which applies the weights of input to 528 predict the true regulatory relationships and delivers the final output of the network as 529 represented by probability for each pair of genes for prediction. To identify a model with 530 a high performance for the prediction, we tried multiple loss functions. The mean 
531 squared logarithmic error loss (MSLE) was selected as the loss function.

Inference of GI3-regulated target genes using top-down GGM algorithm

534 The top-down Gaussian graphical model (GGM) algorithm developed earlier (Wei, 535 2019; Lin et al., 2013) was employed to construct a multilayered gene regulatory 536 network (ML-hGRN) mediated by GL3 in two steps, with the dT1, dT2, EV RNA-seq 537 data being used as the input data. Briefly, in the first step, the GL3 downstream genes 538 that responded to the g/3 activation were identified using Fisher's exact test and the 539 probability-based method as we described in our publications mentioned above, and 540 these genes were termed g/3 responsive genes; in the second step, we further identified 541 those that were interfered frequently by GL3 from the g/3 responsive genes through 542 evaluating all triple gene blocks, each consisting of $\mathrm{g} / 3$, defined as $\mathrm{z}$, and two $\mathrm{g} / 3$ 543 responsive genes, defined as $x$ and $y$. If GL3 significantly interfered with the two 544 responsive genes in a triple gene block, the difference $(d)$ between the correlation 545 coefficient, $r_{x y}$ of two responsive genes in expression and the partial correlation

546 coefficient, $r_{x y / z}$, representing the correlation of two g/3 responsive genes conditioning 547 on $g / 3(z)$ should be significant. The null hypothesis $\mathrm{H}_{0}: d=0$ was tested with the 548 multivariate delta method (MacKinnon et al., 2002). If $d$ is significantly different from 0, $549 \mathrm{~g} / 3$ was concluded to interfere with the two responsive genes and their regulatory 550 relationships were recorded once. After all combined triple gene blocks were evaluated, 551 the interference frequency between $g / 3$ and each responsive gene was calculated. In 552 this study, the candidate target genes with at least one inference frequency were 553 considered to be a gene directly regulated by $\mathrm{g} / 3$.

554 The Metabolomics Facility of the Institute of Genetics and Developmental Biology, 555 Chinese Academy of Sciences

\section{ACKNOWLEDGEMENTS}

558 We thank Fengxia Zhang from the Metabolomics Facility of the Institute of Genetics and 559 Developmental Biology at Chinese Academy of Sciences for wax composition analysis, 560 Wei Wang from Eduard Akhunov laboratory and Melinda Dalby from Barbara Valent 561 laboratory at Kansas State University for helping on confocal microscope experiments. 
562 We thank funding support from the US National Science Foundation (awards no.

563 1741090), the USDA's National Institute of Food and Agriculture (award no. 2018-

564 67013-28511), and the Agricultural Science and Technology Innovation Program of

565 Chinese Academy of Agricultural Sciences. This is the contribution number 21-196-J

566 from the Kansas Agricultural Experiment Station.

567

\section{AUTHOR CONTRIBUTIONS}

569 J.Z., M.C., S.P., H.W., F.F.W, and S.L. conceived and designed experiments. M.Z.,

570 Z.P., Y.Q., B.T., Y.C., G.L., H.Z., K.L., H.T., Y.L., and J.Z. performed experiments and

571 collected data. M.Z., Z.P., Y.Q., L.Z., C.H., H.W., and S. L. analyzed data. M.Z., Z.P.,

572 Y.Q., L.Z., Y.L., M.C., S.P., J.Z., H.W., F.F.W., and S.L. wrote the manuscript with

573 comments from other authors.

574

575 DATA AVAILABILITY

576 Raw dTALe RNA-Seq data are available at NCBI SRA under the project of

577 PRJNA692729.

\section{SUPPLEMENTARY INFORMATION}

580 Supplemental Figures: Supplemental Figures 1-5

581 Supplemental Tables: Supplemental Tables 1-3

582 Supplemental Data Set 1: Detailed information of DEGs

583 Supplemental Data Set 2: Deep learning classification for GL3 downstream genes and

584 dTALe unaffected genes

585 Supplemental Data Set 3: Analyzing result from the top-down GGM of gl3 downstream

586 genes

587 Supplemental Data Set 4: List of 739 B73 RNA-Seq data accessions used for deep

588 learning

589

590 


\section{Parsed Citations}

Aharoni, A, Dixit, S., Jetter, R., Thoenes, E., van Arkel, G., and Pereira, A (2004). The SHINE clade of AP2 domain transcription factors activates wax biosynthesis, alters cuticle properties, and confers drought tolerance when overexpressed in Arabidopsis. Plant Cell 16: 2463-2480.

Google Scholar: Author Only Title Only Author and Title

Antony, G., Zhou, J., Huang, S., Li, T., Liu, B., White, F., and Yang, B. (2010). Rice xa13 recessive resistance to bacterial blight is defeated by induction of the disease susceptibility gene Os-11N3. Plant Cell 22: 3864-3876.

Google Scholar: Author Only Title Only Author and Title

Bartlett, A, O'Malley, R.C., Huang, S.-S.C., Galli, M., Nery, J.R., Gallavotti, A, and Ecker, J.R. (2017). Mapping genome-wide transcription-factor binding sites using DAP-seq. Nat. Protoc. 12: 1659-1672.

Google Scholar: Author Only Title Only Author and Title

Benjamini, Y. and Hochberg, Y. (1995). Controlling the false discovery rate: a practical and powerful approach to multiple testing. J. R.

Stat. Soc. Series B Stat. Methodol. 57: 289-300.

Google Scholar: Author Only Title Only Author and Title

Block, A, Li, G., Fu, ZQ., and Afano, J.R. (2008). Phytopathogen type III effector weaponry and their plant targets. Curr. Opin. Plant Biol. 11: 396-403.

Google Scholar: Author Only Title Only Author and Title

Boch, J., Scholze, H., Schornack, S., Landgraf, A, Hahn, S., Kay, S., Lahaye, T., Nickstadt, A, and Bonas, U. (2009). Breaking the code of DNA binding specificity of TAL-type III effectors. Science 326: 1509-1512.

Google Scholar: Author Only Title Only Author and Title

Bolger, AM., Lohse, M., and Usadel, B. (2014). Trimmomatic: a flexible trimmer for Illumina sequence data. Bioinformatics 30: 21142120.

Google Scholar: Author Only Title Only Author and Title

Büttner, D. and Bonas, U. (2010). Regulation and secretion of Xanthomonas virulence factors. FEMS Microbiol. Rev. 34: 107-133.

Google Scholar: Author Only Title Only Author and Title

Cermak, T., Doyle, E.L., Christian, M., Wang, L., Zhang, Y., Schmidt, C., Baller, J.A, Somia, N.V., Bogdanove, AJ., and Voytas, D.F. (2011). Efficient design and assembly of custom TALEN and other TAL effector-based constructs for DNA targeting. Nucleic Acids Res. 39: e82.

Google Scholar: Author Only Title Only Author and Title

Chen, X. et al. (2017). IRREGULAR POLLEN EXINE1 Is a novel factor in anther cuticle and pollen exine formation. Plant Physiol. 173: 307-325.

Google Scholar: Author Only Title Only Author and Title

Costa, T.R.D., Felisberto-Rodrigues, C., Meir, A, Prevost, M.S., Redzej, A, Trokter, M., and Waksman, G. (2015). Secretion systems in Gram-negative bacteria: structural and mechanistic insights. Nat. Rev. Microbiol. 13: 343-359.

Google Scholar: Author Only Title Only Author and Title

Cunningham, F.J., Goh, N.S., Demirer, G.S., Matos, J.L., and Landry, M.P. (2018). Nanoparticle-mediated delivery towards advancing plant genetic engineering. Trends Biotechnol. 36: 882-897.

Google Scholar: Author Only Title Only Author and Title

Demirer, G.S., Zhang, H., Goh, N.S., Pinals, R.L., Chang, R., and Landry, M.P. (2020). Carbon nanocarriers deliver siRNA to intact plant cells for efficient gene knockdown. Sci Adv 6: eaaz0495.

Google Scholar: Author Only Title Only Author and Title

Deslandes, L. and Rivas, S. (2012). Catch me if you can: bacterial effectors and plant targets. Trends Plant Sci. 17: 644-655.

Google Scholar: Author Only Title Only Author and Title

Dobin, A, Davis, C.A, Schlesinger, F., Drenkow, J., Zaleski, C., Jha, S., Batut, P., Chaisson, M., and Gingeras, T.R. (2013). STAR: ultrafast universal RNAseq aligner. Bioinformatics 29: 15-21.

Google Scholar: Author Only Title Only Author and Title

Fehling, E. and Mukherjee, K.D. (1991). Acyl-CoAelongase from a higher plant (Lunaria annua): metabolic intermediates of very-longchain acyl-CoA products and substrate specificity. Biochim Biophys. Acta 1082: 239-246.

Google Scholar: Author Only Title Only Author and Title

Gleba, Y.Y., Tusé, D., and Giritch, A (2014). Plant viral vectors for delivery by Agrobacterium Curr. Top. Microbiol. Immunol. 375: 155192.

Google Scholar: Author Only Title Only Author and Title

Green, E.R. and Mecsas, J. (2016). Bacterial secretion systems: an overview. Microbiol. Spectr. 4: 1-32.

Google Scholar: Author Only Title Only Author and Title 

available under aCC-BY-ND 4.0 International license.

Hansen, J.D., Pyee, J., Xia, Y., Wen, T.J., Robertson, D.S., Kolattukudy, P.E., Nikolau, B.J., and Schnable, P.S. (1997). The glossy1 Locus of maize and an epidermis-specific cDNA from Kleinia odora Define a class of receptor-like proteins required for the normal accumulation of cuticular waxes. Plant Physiol. 113: 1091-1100.

Google Scholar: Author Only Title Only Author and Title

Hu, Y., Zhang, J., Jia, H., Sosso, D., Li, T., Frommer, W.B., Yang, B., White, F.F., Wang, N., and Jones, J.B. (2014). Lateral organ boundaries 1 is a disease susceptibility gene for citrus bacterial canker disease. Proc. Nat. Acad. Sci. U. S. A111: E521-E529.

Google Scholar: Author Only Title Only Author and Title

Jiao, Y. et al. (2017). Improved maize reference genome with single-molecule technologies. Nature 546: 524-527.

Google Scholar: Author Only Title Only Author and Title

Joung, J.K. and Sander, J.D. (2013). TALENs: a widely applicable technology for targeted genome editing. Nat. Rev. Mol. Cell Biol. 14: 49-55.

Google Scholar: Author Only Title Only Author and Title

Kay, S., Hahn, S., Marois, E., Hause, G., and Bonas, U. (2007). A bacterial effector acts as a plant transcription factor and induces a cell size regulator. Science 318: 648-651.

Google Scholar: Author Only Title Only Author and Title

Khang, C.H., Berruyer, R., Giraldo, M.C., Kankanala, P., Park, S.-Y., Czymmek, K., Kang, S., and Valent, B. (2010). Translocation of Magnaporthe oryzae effectors into rice cells and their subsequent cell-to-cell movement. Plant Cell 22: 1388-1403.

Google Scholar: Author Only Title Only Author and Title

Kunst, L. and Samuels, AL. (2003). Biosynthesis and secretion of plant cuticular wax. Prog. Lipid Res. 42: 51-80.

Google Scholar: Author Only Title Only Author and Title

Lai, X., Stigliani, A, Vachon, G., Carles, C., Smaczniak, C., Zubieta, C., Kaufmann, K., and Parcy, F. (2019). Building transcription factor binding site models to understand gene regulation in plants. Mol. Plant 12: 743-763.

Google Scholar: Author Only Title Only Author and Title

Lee, S.B. and Suh, M.C. (2013). Recent advances in cuticular wax biosynthesis and its regulation in Arabidopsis. Mol. Plant 6: $246-249$. Google Scholar: Author Only Title Only Author and Title

Li, L., Du, Y., He, C., Dietrich, C.R., Li, J., Ma, X., Wang, R., Liu, Q., Liu, S., Wang, G., and Others (2019). Maize glossy6 is involved in cuticular wax deposition and drought tolerance. J. Exp. Bot. 70: 3089-3099.

Google Scholar: Author Only Title Only Author and Title

Li, L., Li, D., Liu, S., Ma, X., Dietrich, C.R., Hu, H.-C., Zhang, G., Liu, Z, Zheng, J., Wang, G., and Schnable, P.S. (2013a). The maize glossy13 gene, cloned via BSR-Seq and Seq-walking encodes a putative ABC transporter required for the normal accumulation of epicuticular waxes. PLoS One 8: e82333.

Google Scholar: Author Only Title Only Author and Title

Lin, Y.C., Li, W., Sun, Y.H., Kumari, S., Wei, H., and Li, Q. (2013). SND1 transcription factor-directed quantitative functional hierarchical genetic regulatory network in wood formation in Populus trichocarpa. The Plant 25: 4324-4341.

Google Scholar: Author Only Title Only Author and Title

Li, T., Huang, S., Zhou, J., and Yang, B. (2013b). Designer TAL effectors induce disease susceptibility and resistance to Xanthomonas oryzae pv. oryzae in rice. Mol. Plant 6: 781-789.

Google Scholar: Author Only Title Only Author and Title

Liu, S., Dietrich, C.R., and Schnable, P.S. (2009). DLA-based strategies for cloning insertion mutants: cloning the gl4 locus of maize using Mu transposon tagged alleles. Genetics 183: 1215-1225.

Google Scholar: Author Only Title Only Author and Title

Liu, S., Yeh, C.-T., Tang, H.M., Nettleton, D., and Schnable, P.S. (2012). Gene mapping via bulked segregant RNASeq (BSR-Seq). PLoS One 7: e36406.

Google Scholar: Author Only Title Only Author and Title

Lu, X. et al. (2018). Gene-indexed mutations in maize. Mol. Plant 11: 496-504.

Google Scholar: Author Only Title Only Author and Title

MacKinnon, D.P., Lockwood, C.M., Hoffman, J.M., West, S.G., and Sheets, V. (2002). Acomparison of methods to test mediation and other intervening variable effects. Psychol Methods 7: 83-104.

Google Scholar: Author Only Title Only Author and Title

Minsavage, G.V. (1990). Gene-for-Gene relationships specifying disease resistance in Xanthomonas campestris pv. vesicatoria Pepper Interactions. Mol. Plant Microbe Interact. 3: 41-47.

Google Scholar: Author Only Title Only Author and Title

Moose, S.P. and Sisco, P.H. (1996). Glossy15, an APETALA2-like gene from maize that regulates leaf epidermal cell identity. Genes Dev. 10: 3018-3027.

Google Scholar: Author Only Title Only Author and Title 
Moscou, M.J. and Bogdanove, AJ. (2009). A simple cipher governs DNA recognition by TAL effectors. Science 326: 1501.

Google Scholar: Author Only Title Only Author and Title

Peng, Z, Hu, Y., Xie, J., Potnis, N., Akhunova, A, Jones, J., Liu, Z, White, F.F., and Liu, S. (2016). Long read and single molecule DNA sequencing simplifies genome assembly and TAL effector gene analysis of Xanthomonas translucens. BMC Genomics 17: 21.

Google Scholar: Author Only Title Only Author and Title

Peng, Z, Hu, Y., Zhang, J., Huguet-Tapia, J.C., Block, AK., Park, S., Sapkota, S., Liu, Z, Liu, S., and White, F.F. (2019). Xanthomonas translucens commandeers the host rate-limiting step in ABA biosynthesis for disease susceptibility. Proc. Natl. Acad. Sci. U. S. A 116: 20938-20946.

Google Scholar: Author Only Title Only Author and Title

Perez-Quintero, AL. et al. (2020). Genomic acquisitions in emerging populations of Xanthomonas vasicola pv. vasculorum Infecting corn in the United States and Argentina. Phytopathology 110: 1161-1173.

Google Scholar: Author Only Title Only Author and Title

Sugio, A, Yang, B., Zhu, T., and White, F.F. (2007). Two type III effector genes of Xanthomonas oryzae pv. oryzae control the induction of the host genes OsTFIIA1 and OsTFX1 during bacterial blight of rice. Proceedings of the National Academy of Sciences 104: 1072010725.

Google Scholar: Author Only Title Only Author and Title

Tacke, E., Korfhage, C., Michel, D., Maddaloni, M., Motto, M., Lanzini, S., Salamini, F., and Döring, H.P. (1995). Transposon tagging of the maize Glossy2 locus with the transposable element En/Spm. Plant J. 8: 907-917.

Google Scholar: Author Only Title Only Author and Title

Tang, J., Yang, X., Xiao, C., Li, J., Chen, Y., Li, R., Li, S., Lü, S., and Hu, H. (2020). GDSL lipase Occluded Stomatal Pore 1 is required for wax biosynthesis and stomatal cuticular ledge formation. New Phytol. 228: 1880-1896.

Google Scholar: Author Only Title Only Author and Title

Tran, T.T. et al. (2018). Functional analysis of African Xanthomonas oryzae pv. oryzae TALomes reveals a new susceptibility gene in bacterial leaf blight of rice. PLoS Pathog. 14: e1007092.

Google Scholar: Author Only Title Only Author and Title

Van den Ackerveken, G., Marois, E., and Bonas, U. (1996). Recognition of the bacterial avirulence protein AvrBs3 occurs inside the host plant cell. Cell 87: 1307-1316.

Google Scholar: Author Only Title Only Author and Title

Wei, H. (2019). Construction of a hierarchical gene regulatory network centered around a transcription factor. Brief. Bioinform 20: $1021-1031$.

Google Scholar: Author Only Title Only Author and Title

Wei, M. et al. (2020). PuHox52-mediated hierarchical multilayered gene regulatory network promotes adventitious root formation in Populus ussuriensis. New Phytol. 228: 1369-1385.

Google Scholar: Author Only Title Only Author and Title

White, F.F., Potnis, N., Jones, J.B., and Koebnik, R. (2009). The type III effectors of Xanthomonas. Mol. Plant Pathol. $10: 749-766$. Google Scholar: Author Only Title Only Author and Title

Xu, X., Dietrich, C.R., Delledonne, M., Xia, Y., Wen, T.J., Robertson, D.S., Nikolau, B.J., and Schnable, P.S. (1997). Sequence analysis of the cloned glossy8 gene of maize suggests that it may code for a [beta]-ketoacyl reductase required for the biosynthesis of cuticular waxes. Plant Physiol. 115: 501-510.

Google Scholar: Author Only Title Only Author and Title

Yang, B., Zhu, W., Johnson, L.B., and White, F.F. (2000). The virulence factor AvrXa7 of Xanthomonas oryzae pv. oryzae is a type III secretion pathway-dependent nuclear-localized double-stranded DNA-binding protein. Proc. Natl. Acad. Sci. U. S. A $97: 9807-9812$. Google Scholar: Author Only Title Only Author and Title

Yilmaz, A, Mejia-Guerra, M.K., Kurz, K., Liang, X., Welch, L., and Grotewold, E. (2011). AGRIS: the Arabidopsis Gene Regulatory Information Server, an update. Nucleic Acids Res. 39: D1118-1122.

Google Scholar: Author Only Title Only Author and Title

Zheng, J., He, C., Qin, Y., Lin, G., Park, W.D., Sun, M., Li, J., Lu, X., Zhang, C., Yeh, C.-T., and Others (2019). Co-expression analysis aids in the identification of genes in the cuticular wax pathway in maize. Plant J. 97: 530-542.

Google Scholar: Author Only Title Only Author and Title

Zhou, J., Peng, Z, Long, J., Sosso, D., Liu, B., Eom, J.-S., Huang, S., Liu, S., Vera Cruz, C., Frommer, W.B., White, F.F., and Yang, B. (2015). Gene targeting by the TAL effector PthXo2 reveals cryptic resistance gene for bacterial blight of rice. Plant J. $82: 632-643$.

Google Scholar: Author Only Title Only Author and Title

Zhu, W., Yang, B., Chittoor, J.M., Johnson, L.B., and White, F.F. (1998). AvrXa10 contains an acidic transcriptional activation domain in the functionally conserved C terminus. Mol. Plant. Microbe. Interact. 11: 824-832.

Google Scholar: Author Only Title Only Author and Title 